\title{
EDITORIAL
}

\section{COPD, asthma and C-reactive protein}

\section{G.P. Anderson}

I $\mathrm{n}$ this issue of the European Respiratory Journal (ERJ), two clinical papers present descriptive reports of the adverse associations between serum C-reactive protein (CRP) levels and indices of disease in chronic obstructive pulmonary disease (COPD) and asthma.

In a Spanish-USA collaboration, DE TORRES et al. [1] report that CRP is elevated in clinically stable COPD patients who had not experienced exacerbations within 2 months, particularly current smokers. They measured Global Initiative for Chronic Obstructive Lung Disease (GOLD) stage, lung function and body composition indices, and reported that elevated CRP levels are negatively correlated with lung function (forced expiratory volume in one second (FEV1), FEV1 per cent predicted (\% pred), forced vital capacity (FVC), FVC \% pred, inspiratory capacity/total lung capacity, particularly, with reduced arterial oxygen tension and poor performance in a 6-min walk distance test. However, current glucocorticosteroid therapy, body composition or cardiovascular comorbidity did not influence CRP levels. In a smaller study, TAKEMURA et al. [2] reported that elevated CRP is negatively associated with lung function and positively associated with sputum eosinophilia in steroid-naive asthmatics, but not in those treated with steroids. The data confirm other clinical findings, but, in the case of COPD, they are discordant for steroid responsiveness, relationship to body mass and lean body mass, and for underlying cardiovascular disease. These and other clinical issues are dealt with in another accompanying editorial by WOUTERS [3].

Elevated CRP has been known for some time to be a predictor of adverse events in cardiovascular disease and is increasingly used as a surrogate marker of systemic inflammation in diverse conditions. The association of elevated CRP with poor lung function indices was first observed a few years ago in population surveys [4]. Similarly, MAN et al. [5] in the USA, SCHOls et al. [6], DENTENER et al. [7] and BROEKHUIZEN et al. [8] in the Netherlands (as early as the mid-1990s) and others have highlighted CRP in COPD, its reduction by glucocorticosteroids and the possibility that this reduction might reduce cardiovascular events, which are frequent in COPD patients [9, 10]. The data presented in the articles in this issue of the ERJ add to the growing literature suggesting that CRP may be a useful systemic biomarker marker of lung inflammation. Since CRP is so easily measured, in comparison to exhaled or more exotic biomarkers, this is good news for researchers. The

CORRESPONDENCE: G.P. Anderson, Lung Disease Research Group, Depts of Medicine and Pharmacology, Chronic Research Centre for Chronic Inflammatory Diseases, The University of Melbourne, Melbourne, Australia. Fax: 61 382440241. E-mail: gpa@unimelb.edu.au articles also raise some difficult and intriguing new scientific questions.

CRP is a member of an ancient family of molecules called the pentraxins, which have been highly conserved in both invertebrates and vertebrates for $>400$ million yrs. It was discovered in humans in 1930 as a serum component that binds the C polysaccharide of Streptococcus pneumoniae (hence CRP). Structurally, CRP is usually composed of five identical subunits (hence pentraxin), each $\sim 23 \mathrm{kDa}$ in mass, which are linked noncovalently to form a disc-like pentagonal ring. For enthusiasts, the CRP gene is on human chromosome 1 at 1q2123. The CRP gene promoter has one intron spacing two coding regions and requires the coordinated action of several transcription factors (CCAAT enhancer binding protein $\beta$ and $\delta$, signal transducer and activator of transcription 3 and nuclear factor (NF)- $\kappa \mathrm{B} /$ Rel proteins) for maximal production. Polymorphisms in the CRP promoter are associated with serum levels [11].

CRP is also a member of a diverse class of defence molecules called the acute-phase proteins, which includes structurally unrelated mannose-binding protein and fibrinogen. Levels of acute-phase proteins rise rapidly, and often dramatically, during infection and after injury; in that case, CRP levels can increase well over 1,000-fold in serious infection when spillover of inflammatory mediators into the blood, especially interleukin (IL)- 6 and IL-1 $\beta$, triggers its production in the liver. It should also be noted that nonhepatic production of CRP by monocytes and lymphocytes has been demonstrated and it is possible (as with leptin [12]) that some CRP is made locally in the inflamed lung, an issue that could be quickly resolved immunohistochemically.

In biology, when a molecule like CRP is both ancient and highly conserved across species, it almost invariably points to function(s) that are essential to preserve life rather than cause disease. CRP unequivocally has beneficial roles; it functions as an opsin to agglutinate bacteria and promote phagocytosis and is directly bacteriocidal. Exogenous CRP protects mice, which oddly are naturally deficient in CRP, from diverse lethal infections. CRP is an excellent scavenger of chromatin from damaged cells, such as those that abound in the apoptosisprone COPD lung, which would otherwise cause severe damage to nearby tissue. In other studies, CRP has been shown to induce anti-inflammatory IL-1 decoy receptors, blunt the shedding of L-selectin that precedes the movement of neutrophils from blood to tissue and to dampen leukocyte oxidative burst. Recent studies also suggest that CRP can trigger anti-inflammatory immunoglobulin crystallisable fragments gamma $(\mathrm{Fc} \gamma)$ inhibitory receptors bearing a 
cytoplasmic immunoreceptor tyrosine-based inhibitory motif. Is the negative correlation of CRP with COPD, asthma (and indeed several other inflammatory diseases), therefore, a paradox? This is most unlikely.

CRP can also act as a potent pro-inflammatory agent. CRP activates the classical complement cascade, which can be intensely pro-inflammatory, by binding directly to the complement fragment C1q. CRP also activates NF- $\kappa B$ in endothelial cells and mononuclear cells to induce proteases and proinflammatory cytokines, such as IL-1 $\beta$, IL-6, IL-8 and IL-18 [13]. Interestingly, especially in the context of the vascular apoptosis theory of emphysema, CRP represses the repair potential of endothelial stem cells [14]. Considering the negative lung function associations of elevated CRP, this warrants further study.

One of the most vexing questions raised by the articles in this issue of the ERJ is what stimulates CRP production in clinically stable disease? CRP is classically induced on infection. Although the lungs could be expected to be colonised in $\sim 30 \%$ of the COPD patients in the study by DE TORRES et al. [1] (the authors do not report on this), and perhaps a few of the patients in the study by TAKEMURA et al. [2] might have Chlamydia or Mycoplasma infection (no data here either), it is most unlikely that smouldering lung infection was the actual cause of elevated CRP. Other infections also not studied, including subchronic mycobacteria, adenovirus and cytomegalovirus (CMV), as well as periodontal disease, could be drivers. Particulates in polluted air might also contribute [15]. It could also be because some of the patients were obese, since adipocytes release IL- 6 and can induce CRP, but this seems unlikely based on the presented data. Other comorbidities that are known to raise CRP were specifically excluded from the studies.

One key to understanding this problem may lie in the kinetics of innate immune defence reactions. CRP, like other innate immunity effector molecules, is part of a stereotypical and very rapid response that uses inflammation to kill or frustrate invading pathogens and contain tissue injury. The proinflammatory activity of CRP is clearly beneficial in this context. When the danger has passed and the pathogen is killed, the primary stimulus is removed. Danger signals sent by pattern recognition receptors (such as the Toll-like receptor (TLR)2), which innately recognise pathogen-associated molecular patterns (such as lipotechoic acid in S. pneumoniae C polysaccharide), cease and the levels of these effectors fall rapidly to the baseline (CRP is normally low or absent in health). Sustained levels of innate immunity defence molecules (such as CRP, high-mobility group box protein 1, receptor for advanced glycosylation end-product (RAGE) ligands and others) are increasingly being recognised as pathogenic in diverse diseases, where it is thought that the low-level inflammation they cause contributes to progressive tissue and functional attrition in multiple organs.

As elevated CRP was found in stable asthma and ex-smokers with COPD, it seems unavoidable to conclude that diseased lung tissue itself must provide the sustained and unrelenting "danger" signal that drives CRP synthesis. This could be because damaged lung tissue might generate endogenous TLR ligands, such as fibronectin, or heat shock proteins, triggering direct inflammation. This could be further amplified by persistent somatic mutations in regulatory genes, such as epidermal growth factor receptor, ras oncogene and phosphatase and tensin homologue deleted on chromosome 10 that abound in smoke-exposed epithelium [16], and by transcriptional dysregulation secondary to loss of histone deacetylases [17], especially in more severe disease. Understanding exactly why CRP is elevated in stable disease may provide answers to other difficult problems, such as why smoking cessation does not reverse lung inflammation in COPD and why steroids can suppress but not cure asthma.

This much seems certain: IL- 6 and IL- $1 \beta$ are directly implicated since CRP is principally induced by IL- 6 and this is amplified by IL- 1 . Both IL- 6 and IL- $1 \beta$ have been found in asthma and COPD [8, 18]. DE TORRES et al. [1] reported the highest CRP levels in current smokers and it is known that cigarette smoke induces IL-6 in lung tissue, but not lung macrophages, in vivo [19]. IL-6 was not measured in either of the studies appearing in this issue of the ERJ [1, 2], but recent reports show it is unequivocally elevated in COPD and follows a pattern of association with disease severity and morbidity [8], similar to the pattern DE TORRES et al. [1] described in their article. Indeed, IL-6 may be the major, if cryptic, message of these studies, at least for COPD. IL-6 is not just the primary inducer of CRP and other acute-phase proteins; it is also a potent inducer of skeletal muscle wasting [20], bone loss [21] and probably increased chest infection risk [22], which are major disease comorbidities in COPD. Since clinical-grade monoclonal antibodies against human IL-6 have already been developed, a clinical trial of the effect of anti-IL-6 in COPD seems obvious; CRP could even serve as a surrogate marker.

The CRP infection link cannot be ignored because CRP spikes during acute exacerbations of COPD. Since exacerbations have been linked to accelerated and stepwise decline in lung function and worsening of COPD comorbidities, including wasting, it will be very import to understand the relationship between CRP and disease progression in longitudinal studies.

In contrast to other studies, DE TORRES et al. [1] found no obvious association with underlying cardiovascular disease; this is perhaps not surprising in a relatively small $(\sim 200$ including controls) cross-sectional study. Large prospective population cohort studies are now needed to weight the meaning of CRP in COPD. However, the current ERJ articles $[1,2]$ do raise the issue of whether CRP will produce the same risks in asthma and COPD. While poor cardiovascular health and increased event rate are well documented in COPD, this is not currently thought to be the case in asthma. This may change in the future as asthma is now becoming more associated with obesity and many asthmatics smoke. It should be noted that the levels of CRP were lower in asthma [2] than COPD [1]. Careful epidemiological studies are needed.

It is even possible that CRP may behave differently in asthma and COPD. CRP is most damaging when it is aggregated or altered. One of the most important themes emerging in COPD research is that smoking, and probably the disease itself, chemically alters multiple tissue and intracellular proteins in the lung and systemically, for example, by oxidation. Not only 
will the amount of CRP that is present need to be ascertained, but also in what form it occurs; this may be particularly important if CRP turns out to be produced locally in the lung as well as by the liver. Detection of modification is now relatively straightforward using proteomics methods.

Several recent studies have suggested that inhaled steroids can reduce systemic $\mathrm{C}$-reactive protein in chronic obstructive pulmonary disease (the C-reactive protein effect in asthma seems very steroid sensitive based on TAKEMURA et al. [2]) and that this may be linked with decreased mortality [10, 23]. However, DE TORRES et al. [1] found no clear effect. All groups could be correct: the effect may be small or restricted to patient subgroup(s). Given that inhaled steroids, often in combination products with long-acting $\beta$-agonists, are now widely used in chronic obstructive pulmonary disease and very large prospective studies, sponsored by their manufacturers, are underway [24], it will be extremely important to weigh this much awaited data cautiously and critically.

\section{REFERENCES}

1 de Torres JP, Cordoba-Lanus E, López-Aguilar C, et al. C-reactive protein levels and clinically important predictive outcomes in stable COPD patients. Eur Respir J 2006; 27: 902-907.

2 Takemura A, Matsumoto H, Niimi A, et al. High sensitivity C-reactive protein in asthma. Eur Respir J 2006; 27: 908-912.

3 Wouters EFM. The systemic face of airway diseases: the role of C-reactive protein. Eur Respir J 2006; 27: 877-879.

4 Kony S, Zureik M, Driss F, Neukirch C, Leynaert B, Neukirch F. Association of bronchial hyperresponsiveness and lung function with C-reactive protein (CRP): a population based study. Thorax 2004; 59: 892-896.

5 Man SFP, Sin DD. Effects of corticosteroids on systemic inflammation in chronic obstructive pulmonary disease. Proc Am Thorac Soc 2005; 2: 78-82.

6 Schols AM, Buurman WA, Staal van den Brekel AJ, Dentener MA, Wouters EF. Evidence for a relation between metabolic derangements and increased levels of inflammatory mediators in a subgroup of patients with chronic obstructive pulmonary disease. Thorax 1996; 51: 819-824.

7 Dentener MA, Creutzberg EC, Schols AM, et al. Systemic anti-inflammatory mediators in COPD: increase in soluble interleukin 1 receptor II during treatment of exacerbations. Thorax 2001; 56: 721-726.

8 Broekhuizen R, Wouters EF, Creutzberg EC, Schols AM. Raised CRP levels mark metabolic and functional impairment in advanced COPD. Thorax 2006; 61: 17-22.

9 Gan WQ, Man SFP, Sin DD. The interactions between cigarette smoking and reduced lung function on systemic inflammation. Chest 2005; 127: 558-564.
10 Sin DD, Wu L, Anderson JA, et al. Inhaled corticosteroids and mortality in chronic obstructive pulmonary disease. Thorax 2005; 60: 992-997.

11 Carlson CS, Aldred SF, Lee PK, et al. Polymorphisms within the C-reactive protein (CRP) promoter region are associated with plasma CRP levels. Am J Hum Genet 2005; 77: 64-77.

12 Broekhuizen R, Vernooy JH, Schols AM, Dentener MA, Wouters EF. Leptin as local inflammatory marker in COPD. Respir Med 2005; 99: 70-74.

13 Ballou SP, Lozanski G. Induction of inflammatory cytokine release from cultured human monocytes by C-reactive protein. Cytokine 1992; 4: 361-368.

14 Suh W, Kim KL, Choi JH, et al. C-reactive protein impairs angiogenic functions and decreases the secretion of arteriogenic chemo-cytokines in human endothelial progenitor cells. Biochem Biophys Res Commun 2004; 321: 65-71.

15 van Eeden SF, Yeung A, Quinlam K, Hogg JC. Systemic response to ambient particulate matter: relevance to chronic obstructive pulmonary disease. Proc Am Thorac Soc 2005; 2: 61-67.

16 Anderson GP, Bozinovski S. Acquired somatic mutations in the molecular pathogenesis of COPD. Trends Pharmacol Sci 2003; 24: 71-76.

17 Ito $\mathrm{K}$, Ito $\mathrm{M}$, Elliott $\mathrm{WM}$, et al. Decreased histone deacetylase activity in chronic obstructive pulmonary disease. N Engl J Med 2005; 352: 1967-1976.

18 Doganci A, Sauer K, Karwot R, Finotto S. Pathological role of IL-6 in the experimental allergic bronchial asthma in mice. Clin Rev Allergy Immunol 2005; 28: 257-270.

19 Vlahos R, Bozinovski S, Jones JE, et al. Differential protease, innate immunity and $\mathrm{NF \kappa B}$ induction profiles during lung inflammation induced by sub-chronic cigarette smoke exposure in mice. Am J Physiol Lung Cell Mol Physiol 2005; Epub ahead of print PMID: 16361358.

20 Hansen MJ, Gualano RC, Bozinovski S, Vlahos R, Anderson GP. Therapeutic prospects to treat skeletal muscle wasting in COPD (chronic obstructive lung disease). Pharmacol Ther 2006; 109: 162-172.

21 Scheidt-Nave C, Bismar H, Leidig-Bruckner G, et al. Serum interleukin 6 is a major predictor of bone loss in women specific to the first decade past menopause. J Clin Endocrinol Metab 2001; 86: 2032-2042.

22 Yende S, Tuomanen EI, Wunderink R, et al. Preinfection systemic inflammatory markers and risk of hospitalization due to pneumonia. Am J Respir Crit Care Med 2005; 172: 1440-1446.

23 Sin DD, Lacy P, York E, Man SF. Effects of fluticasone on systemic markers of inflammation in chronic obstructive pulmonary disease. Am J Respir Crit Care Med 2004; 170: 760-765.

24 Man SF, Sin DD. Effects of corticosteroids on systemic inflammation in chronic obstructive pulmonary disease. Proc Am Thorac Soc 2005; 2: 78-82. 